\title{
Non-resolvinganemia with gastrointestinal stromal tumors - Case report
}

\author{
Thilakawrdana Basuru Uvindu ${ }^{1 \$}$, De Mel Sanjay ${ }^{1}$, Abesuriya Vasitha ${ }^{2}$, De Mel \\ Chandima ${ }^{1}$, Chandrasena $\mathrm{Lal}^{1}$, And Abesuriya Visula ${ }^{1}$ \\ ${ }^{I}$ Nawaloka Hospitals Research And Educational Centre, Nawaloka Hospitals PLC, No. 23, Deshamanya H.K. \\ Dharmadasa Mw, Colombo 02, Sri Lanka \\ ${ }^{2}$ University Of Kelaniya, Faculty Of Medicine Ragama, Sri Lanka \\ ${ }^{\$}$ Corresponding Author Thilakawrdana Basuru Uvindu, Nawaloka Hospitals Research And Educational Centre, \\ Nawaloka Hospitals PLC, Deshamanya H.K. Dharmadasa Mw, Colombo 02, Sri Lanka
}

\begin{abstract}
Prevalence of non-communicable diseases are higher among the Sri Lankan society.Alarge roportion were carcinomas of different systems. The demographic transition has a pivotal role for this diseases burden. Gastrointestinal stromal tumors (GIST) are among rare clinical entity. At present private health sector in Sri Lanka is capable of detecting these neoplasms. This report presents probably the first case of Jejunal gastrointestinal stromal tumor operated recently in a 56-year-old female patient, in private hospital. The diagnosis, treatment, outcome, as well as literature review of the pathological identification, sites of origin and prognosis as presented as follows.
\end{abstract}

Keywords: Leiomyosarcoma, anaemia, immunohistochemistry, enteroscopy

\section{Introduction}

Gastro Intestinal Stromal Tumorsare rare neoplasms which needs molecular and immunohistochemical basis for early detection ${ }^{[1]}$. These tumors of gastrointestinal tract accounting less than $1 \%$ of all gastro intestinal neoplasms ${ }^{[2]}$. Early detection of these tumors will results in increased number of successful curative surgical interventions which leads to good prognosis. C- KITPROTO-ONCOGENE gain-of-function mutations were detected by Hirota and colleagues in 1998had shown GIST can be diagnosed by using this genetic alteration. This led to identify GIST as a different clinical entity and it was a major step in diagnosis of the disease. ${ }^{[3]}$

\section{Case report}

This patient was 56 year old lady who had clinical symptoms of on and off shortness of breath for nearly 8 months.Clinical history and investigations reviled that she was having symptoms of anemia (shortness of breath, sleepiness and lethargy) with episodic hemoglobin variation between $7-11 \mathrm{mg} / \mathrm{dl}$ and positive for fecal occult blood.Furthermore,history reviled thatshe was asymptomatic while her hemoglobin levels were around $10-11 \mathrm{mg} / \mathrm{dl}$. At this point her fecal matter was not positive for occult blood. A clinical co-relation was observed that she was symptomatic while her fecal occult blood was positive. The same time biochemical investigations hadreviled her hemoglobin levels were varying around $9-9.5 \mathrm{mg} / \mathrm{dl}$. Despiteshe had several episodes but no constitutional symptoms such as loss of appetite.

She had been investigated upper Gastrointestinal and lower Gastrointestinal endoscopy in several times by different centers. But no abnormality had been detected. No center had been performed a contrast radio imaging studies. Neither our endoscopic interventions which includes upper Gastrointestinal endoscopy up to D3, D4, lower Gastrointestinal endoscopy up to terminal ileum and capsule endoscopyhad not reviled any intra luminal pathologies.

Later she had been referred to a consultant hematologist for further assessment.Hematological investigations including blood picturewas reported thatshe was having iron deficiency anemia. Finally,Enteroscopy was performed by a consultant surgeon. Which detected a midJejunal intra luminal growth with a patched of haemorrhage. The tumor was involving 30-40\% of the lumen of mid Jejunum.

Figure 1 : Findings of enteroscopy in mid Jejunum

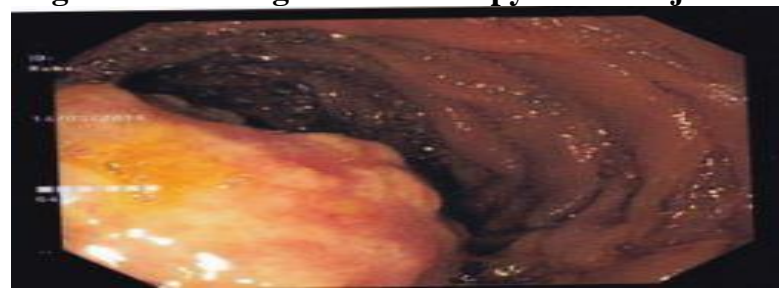




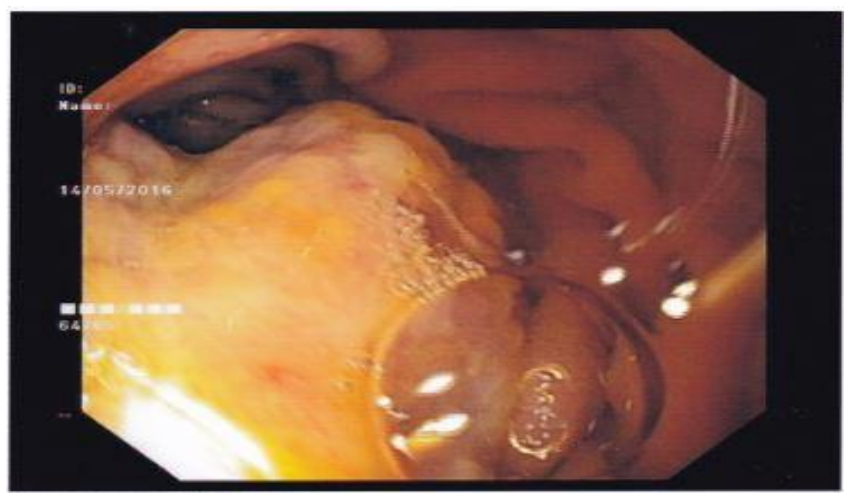

After enteroscopy procedure and its' findings clinical team has decided to proceed with contrast enhanced Computed Tomography(CT)abdomen scan (IV and oral) to further evaluate the lesion and to plan the surgery. CT was performed 640 Slice Computed Tomography (CT) scan (Aquilion one edition, TOSHIBA 2012).

Report of the Contrast Enhanced CT abdomen scan indicated that there is a $30 \mathrm{~mm} \times 26 \mathrm{~mm}$ well defined mass in one of the proximal Jejunal loop of bowel and it did not enhance with the contrast. Oral contrast was seen passing around the mass and therewas no complete obstruction. No regional lymphadenopathy was identified.

The multi-disciplinary team had thorough discussion with the patient and guardians and explained the clinical findings, lab investigation reports and findings of interventions. They also had been explained the treatment modalities, possible outcomes and survivorship. After obtaining informed written consent surgery was planned.The midline laparotomy was performed. The growth was removed with a tumor free margin of more than $5 \mathrm{~cm}$ and side to side Jejunal anastomosis was performed by using 60GIA Boston Scientific Stapler (Echelon Flex ${ }^{\mathrm{TM}}$ Endopath ${ }^{\circledR}$ staplers, Ethicon US, LLC, 2010/2016). The surgically removed specimen was a segment of small bowel measuring $110 \mathrm{~mm}$ in length and $80 \mathrm{~mm}$ in diameter which was sent for histological analysis.

The histopathology report reviles that the polypoid growth was $30 \mathrm{X} 25 \mathrm{X} 15 \mathrm{~mm}$ in size, macroscopically. Histological examination reviled sections of polypoid lesion reveal a circumscribed spindle cell proliferation arising in the muscularis propria. It was composed of interlacing fascicles and haphazardly arranged spindle shaped cells with elongated and plump nuclei. The cells contain eosinophilic cytoplasm. There are scattered, enlarged, pleomorphic nuclei. Vague nuclear palisading was focally present. The mitotic count was 4/50 High Power Field(HPF). There was no tumor necrosis. The tumor extent to the submucosa with a pushing margin, but the mucosa was not involved by the tumor. The small bowel mucosa was histologically unremarkable. In conclusion tumor type was identified as spindle cell tumor possibilities include GIST or smooth muscle tumor.Following immunohistochemical markers were recommended after histological inspection. CD117, DOG-1, SMA, Desmin, S100, Ki-67.

After that Immunohistochemical assay was performed. Itdemonstrated that the tumor cells stain strongly positive with SMA and desmin. They stain negatively with S100 and CD 117. Ki-67 shows a proliferation index between 5 to $10 \%$. In conclusion immunohistochemistry confirms the diagnosis of a smooth muscle tumor, in-view of the scattered nuclear atypia and low-mitotic activity, it is classified as a well differentiated, low grade leiomyosarcoma (FNCLCC grade 1)

\section{Discussion}

The main objective of this study is to emphasize on a rare gastro intestinal pathology which causes chronic anemia and to convince that it can be easily misdiagnosed even with extensive investigations. Incidence in population of Gastro Intestinal Stromal Tumors is about 2: $100000^{[4]}$. The commonest site ofGIST was stomach(60\%),Following duodenum, colorectal region, Oesophaguas and appendix. Studies also highlighted it is extremely rare inJejunum [4][5][6]. Literature showed that tumor size is associated with the final prognosis.It demonstrated that tumor of more that $10 \mathrm{~cm}$ had a poor prognosis due to increased possibility of metastasis[6]. This case report showed that stromal tumor size was $3 \mathrm{~cm}$. furthermore there were no signs of metastasis intraoperatively and histology confirmed that obtained tumor free margins in surgical resection.

During further follow up visits we noted that her haemoglobin level was rising towards normal and her symptoms were resolving. she was considered as a low risk patientbased on Fletcher's scheme of predicting risk $^{[7]}$. This can predict there is a lower risk of metastasis which can leads to a better prognosis. 
Another aspect we would like to demonstrate that GIST is one of the rare but possible differential diagnosis for chronic anemic with bleeding per rectum. In this case bleeding per rectum was not visible bleeding, but demonstrated by positive fecal occult blood in rapid test for human red blood cells. This may be due to early stage of GIST of our case report.

We have performed ultra sound scan to detect any abdominal pathologies in this case. But it has not identified any abnormalities. Studies showed that even an ultrasound scan of the abdomen is often first line choice of imaging study with a patient presents with abdominal pain and a lump ${ }^{[9]}$ furthermore it reviles that it is not very much informative as most of the lumps detected were unable to demonstratedorigin of organ by ultrasound scan. Often ultrasonography findings reviles that foci of necrosis and heterogenous reflectivities in large abdominal masses. Literature suggests that CT provides the fundamental data to diagnose and stage a GIST of most patients. Most number of tumors demonstrates varying density and patchy enhancement after intra venous contrast. Within the mass there could be varying degree of necrotic foci which also can demonstrated in contrast -enhanced CT scan[8]. In contrast to previous studies CT scan report of this case report of this study showed that therewas a mass in the Mid jejunum despite its smaller volume. This may be due to better preparation of the patient and the image quality of the CT scan. The literature reviles that GIST occurs marginally frequent in males as compared to females, both in the fifth and sixth decades of life. There is no racial or geographical preponderance. The patient presented in our case report was 56 years which is compatible to previous study findings, but female sex. ${ }^{[9]}$

\section{Conclusion}

GIST is a rare disease. It is a rare cause for chronic anemia as well. Failure to detect early will case poor prognosis of the patient due to aggressiveness of the disease. Therefore, advocacy and awareness is a must. It needs multidisciplinary approach to obtain successful outcome of the patient.

\section{Acknowledgement}

The authors reported that there was no conflict of interest and no funding was received.

\section{Reference}

[1]. De Silva CM, Reid R. Gastrointestinal stromal tumors (GIST): C-kit mutations, CD117 expression, differential diagnosis and targeted cancer therapy with Imatinib. Pathology and Oncology Research. 2003;9(1):13-9. Epub 2003 Apr 18.

[2]. Cichoz-Lach H, Kasztelan-Szczerbińska B, Słomka M.Gastrointestinal stromal tumors: epidemiology, clinical picture, diagnosis, prognosis and treatment. Polish Archives of Internal Medicine 2008 Apr;118(4):216-21.

[3]. Hirota S, Isozaki K, Moriyama Y, Hashimoto K, Nishida T, Ishiguro S, et al. Gain-of-function mutations of c-kit in human gastrointestinal stromal tumors. Science 1998. Jan;279(5350):577-580 10.1126/science.279.5350.577

[4]. Dhull AK, Kaushal V, Dhankhar R, et al. The Inside Mystery of Jejunal Gastrointestinal Stromal Tumor: A Rare Case Report and Review of the Literature. Case Reports in Oncological Medicine. 2011;2011:1-4.

[5]. Alexander B, Inga-Marie S, Philipp S, et al. Gastrointestinal stromal tumors. International Journalof Colorectal Disease. 2012;27:689-700.

[6]. Takeshi T, Takaaki S, Shinji S, et al. Primary omental Gastrointestinal stromal tumor (GIST) World Journal of Surgical Oncology.2007;5:66-71

[7]. Fletcher CD, Berman JJ, Corless C, et al. Diagnosis of gastrointestinal stromal tumors: A consensus approach. Human Pathology.2002;33:459-465

[8]. King DM. The radiology of gastrointestinal stromal tumours (GIST). Cancer Imaging 2005;5(1):150-156 10.1102/14707330.2005.0109

[9]. Palankezhe S, Apoorva M, Usha M et al. Gastrointestinal Stromal Tumors: A Case Report. Oman Medical Journal. 2014 Mar; 29(2): 138-141. 